\title{
Donor Hemovigilance: Need of the Hour
}

\section{Rajeswari Subramaniyan*}

Department of Transfusion Medicine, Yashoda Hospital, Malakpet, Hyderabad-500036, India

*Corresponding author: Rajeswari Subramaniyan, Department of Transfusion Medicine, Yashoda Hospital, Malakpet, Hyderabad-500036, India, Tel: 040 67777888; Email: arthisoundarya@gmail.com

Received date: Oct 24, 2015, Accepted date: Nov 07, 2015, Publication date: Nov 12, 2015

Copyright: ( 2015 Subramaniyan R. This is an open-access article distributed under the terms of the Creative Commons Attribution License, which permits unrestricted use, distribution, and reproduction in any medium, provided the original author and source are credited.

\begin{abstract}
Delayed off site adverse donor reactions are rare. Here we report a donor who presented with pain and swelling in right shoulder after an off site injury. Evaluation revealed fracture clavicle. These delayed reactions can be mitigated by donor hemovigilance.
\end{abstract}

Keywords: Delayed adverse reactions; Donor hemovigilance

\section{Letter}

A 30-year-old gentleman, a voluntary repeat donor was selected as per the standard operating procedure of the department. His height and weight were $172 \mathrm{~cm}$ and $78 \mathrm{~kg}$ respectively. His predonation parameters were within normal limits and there was no previous history of donor reaction. He donated $450 \mathrm{ml}$ whole blood $(<10 \%$ of the total blood volume, his total blood volume being $4981 \mathrm{ml})$. The donation was uneventful. Twenty minutes after leaving the hospital premise she was brought back with history of fainting followed by fall on the floor. He complained of pain in the right shoulder with inability to lift his arm. On examination, he was conscious and oriented. His pulse was $62 \mathrm{bpm}$ and blood pressure was 100/70 mmHg. Examination of the right shoulder revealed swelling and tenderness. There were no signs of raised intracranial tension. No other associated injuries were observed. Further evaluation revealed fracture in the middle third of clavicle (Figure 1). Orthopedic intervention was made with a figure of eight brace and hospitalised for a day. Fracture union was noted during 6 weeks follow-up. The donor was advised to refrain from donating blood indefinitely. Based on the classification of Working Group on Donor Vigilance of the International Society of Blood Transfusion Working Party on Haemovigilance, this delayed vasovagal reaction is of severem grade (due to hospitalisation and intervention) and definite imputability. Age, gender, weight, total blood volume, first time donor status are known predictors of syncope. The incidence of delayed ( $>15$ minutes) off site reactions is underestimated. Delayed reactions are probably due to orthostatic intolerance (non neurogenic) exacerbated by hypovolemia, though immediate ( $<15$ minutes) reactions are neurocardiogenic (vasovagal) syncope [1]. These reactions can be mitigated by intake of salt and water. Donor hemovigilance should be improved with stringent reporting system to analyse and minimise donor reactions thereby ensuring donor retention and safety.

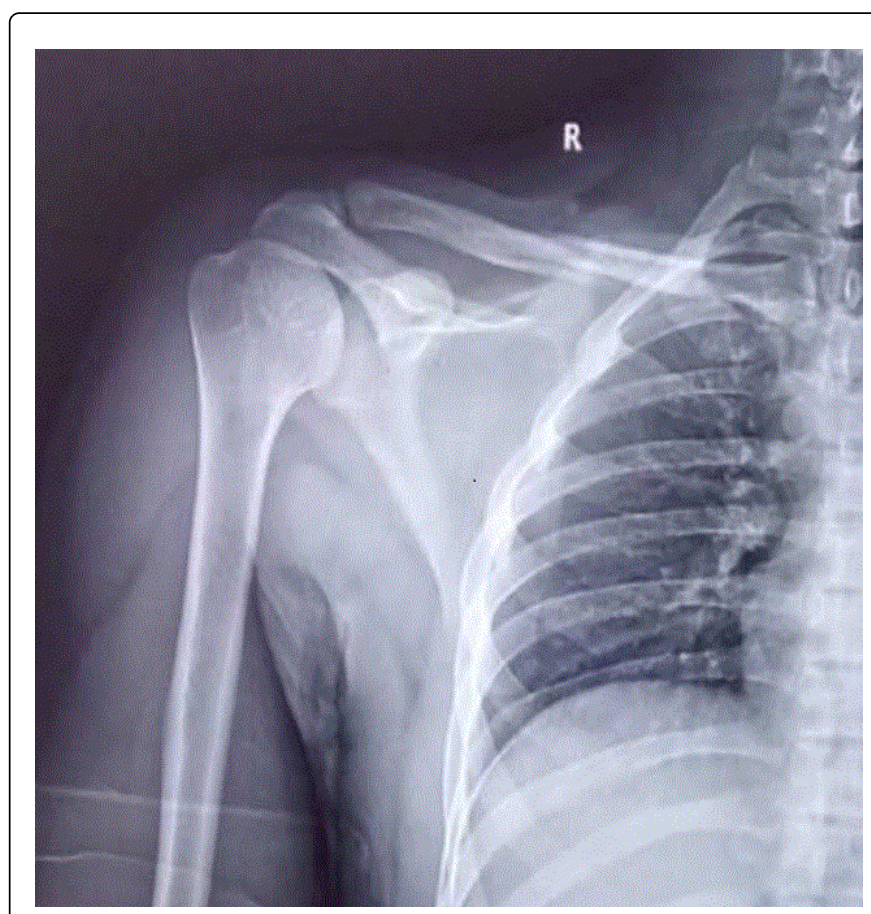

Figure 1: Evaluation revealed fracture in the middle third of clavicle.

\section{References}

1. Kamel H, Tomasulo P, Bravo M, Wiltbank T, Cusick R, et al. (21010) Delayed adverse reactions to blood donation. Transfusion 50: 556-565. 